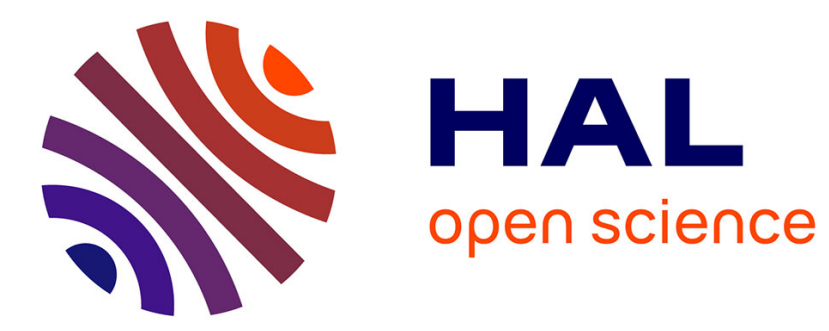

\title{
Three Millisecond Pulsars in FERMI LAT Unassociated Bright Sources
}

S. M. Ransom, P. S. Ray, F. Camilo, M. S. E. Roberts, O. Celik, M. T. Wolff, C. C. Cheung, M. Kerr, T. Pennucci, M. E. Decesar, et al.

\section{> To cite this version:}

S. M. Ransom, P. S. Ray, F. Camilo, M. S. E. Roberts, O. Celik, et al.. Three Millisecond Pulsars in FERMI LAT Unassociated Bright Sources. The Astrophysical journal letters, 2011, 727, pp.L16. 10.1088/2041-8205/727/1/L16 . in2p3-00586142

\section{HAL Id: in2p3-00586142 https://hal.in2p3.fr/in2p3-00586142}

Submitted on 10 Dec 2019

HAL is a multi-disciplinary open access archive for the deposit and dissemination of scientific research documents, whether they are published or not. The documents may come from teaching and research institutions in France or abroad, or from public or private research centers.
L'archive ouverte pluridisciplinaire HAL, est destinée au dépôt et à la diffusion de documents scientifiques de niveau recherche, publiés ou non, émanant des établissements d'enseignement et de recherche français ou étrangers, des laboratoires publics ou privés. 


\title{
THREE MILLISECOND PULSARS IN FERMI LAT UNASSOCIATED BRIGHT SOURCES
}

\author{
S. M. Ransom ${ }^{1}$, P. S. Ray ${ }^{2}$, F. Camilo ${ }^{3}$, M. S. E. Roberts ${ }^{4}$, Ö. Çelik ${ }^{5,6,7}$, M. T. WolfF ${ }^{2}$, C. C. Cheung ${ }^{8,21}$, M. KerR ${ }^{9}$, \\ T. Pennucci ${ }^{10}$, M. E. DeCesar ${ }^{5,11}$, I. Cognard ${ }^{12}$, A. G. Lyne $^{13}$, B. W. Stappers ${ }^{13}$, P. C. C. Freire ${ }^{14}$, J. E. Grove ${ }^{2}$, \\ A. A. Abdo ${ }^{8}$, G. Desvignes ${ }^{15}, 16$, D. Donato ${ }^{6,11}$, E. C. Ferrara ${ }^{5}$, N. Gehrels ${ }^{5}$, L. Gulllemot ${ }^{14}$, C. Gwon $^{2}$, A. K. Harding $^{5}$, \\ S. Johnston $^{17}$, M. Keith ${ }^{17}$, M. Kramer ${ }^{13,14}$, P. F. Michelson ${ }^{9}$, D. Parent ${ }^{18,21}$, P. M. Saz Parkinson ${ }^{19}$, R. W. Romani ${ }^{9}$, \\ D. A. Smith $^{20}$, G. Theureau ${ }^{12}$, D. J. Thompson ${ }^{5}$, P. Weltevrede ${ }^{13}$, K. S. Wood ${ }^{2}$, And M. Ziegler ${ }^{19}$ \\ ${ }^{1}$ National Radio Astronomy Observatory (NRAO), Charlottesville, VA 22903, USA; sransom@nrao.edu \\ ${ }^{2}$ Space Science Division, Naval Research Laboratory, Washington, DC 20375, USA; Paul.Ray@ nrl.navy.mil \\ ${ }^{3}$ Columbia Astrophysics Laboratory, Columbia University, New York, NY 10027, USA \\ ${ }^{4}$ Eureka Scientific, Oakland, CA 94602, USA \\ ${ }^{5}$ NASA Goddard Space Flight Center, Greenbelt, MD 20771, USA \\ ${ }^{6}$ Center for Research and Exploration in Space Science and Technology (CRESST) and NASA Goddard Space Flight Center, Greenbelt, MD 20771, USA \\ ${ }^{7}$ Department of Physics and Center for Space Sciences and Technology, University of Maryland Baltimore County, Baltimore, MD 21250, USA \\ ${ }^{8}$ National Research Council Research Associate, National Academy of Sciences, Washington, DC 20001, USA \\ ${ }^{9}$ W. W. Hansen Experimental Physics Laboratory, Kavli Institute for Particle Astrophysics and Cosmology, Department of Physics and SLAC National Accelerator \\ Laboratory, Stanford University, Stanford, CA 94305, USA \\ ${ }^{10}$ Department of Astronomy, University of Virginia, Charlottesville, VA 22904, USA \\ ${ }^{11}$ Department of Physics and Department of Astronomy, University of Maryland, College Park, MD 20742, USA \\ ${ }^{12}$ Laboratoire de Physique et Chimie de l'Environnement, LPCE UMR 6115 CNRS, F-45071 Orléans Cedex 02, and Station de radioastronomie de Nançay, \\ Observatoire de Paris, CNRS/INSU, F-18330 Nançay, France \\ 13 Jodrell Bank Centre for Astrophysics, School of Physics and Astronomy, The University of Manchester, M13 9PL, UK \\ ${ }_{14}^{14}$ Max-Planck-Institut für Radioastronomie, Auf dem Hügel 69, 53121 Bonn, Germany \\ 15 Department of Astronomy, University of California, Berkeley, CA 94720-3411, USA \\ ${ }^{16}$ Radio Astronomy Laboratory, University of California, Berkeley, CA 94720, USA \\ ${ }^{17}$ CSIRO Astronomy and Space Science, Australia Telescope National Facility, Epping, NSW 1710, Australia \\ ${ }^{18}$ College of Science, George Mason University, Fairfax, VA 22030, USA \\ ${ }^{19}$ Santa Cruz Institute for Particle Physics, Department of Physics and Department of Astronomy and Astrophysics, University of California at Santa Cruz, \\ Santa Cruz, CA 95064, USA \\ ${ }^{20}$ Université Bordeaux 1, CNRS/IN2p3, Centre d'Études Nucléaires de Bordeaux Gradignan, 33175 Gradignan, France \\ Received 2010 August 17; accepted 2010 December 8; published 2010 December 23
}

\begin{abstract}
We searched for radio pulsars in 25 of the non-variable, unassociated sources in the Fermi LAT Bright Source List with the Green Bank Telescope at $820 \mathrm{MHz}$. We report the discovery of three radio and $\gamma$-ray millisecond pulsars (MSPs) from a high Galactic latitude subset of these sources. All of the pulsars are in binary systems, which would have made them virtually impossible to detect in blind $\gamma$-ray pulsation searches. They seem to be relatively normal, nearby ( $\leqslant 2 \mathrm{kpc}$ ) MSPs. These observations, in combination with the Fermi detection of $\gamma$-rays from other known radio MSPs, imply that most, if not all, radio MSPs are efficient $\gamma$-ray producers. The $\gamma$-ray spectra of the pulsars are power law in nature with exponential cutoffs at a few $\mathrm{GeV}$, as has been found with most other pulsars. The MSPs have all been detected as X-ray point sources. Their soft X-ray luminosities of $\sim 10^{30}-10^{31} \mathrm{erg} \mathrm{s}^{-1}$ are typical of the rare radio MSPs seen in X-rays.
\end{abstract}

Key words: pulsars: general - pulsars: individual (J0614-3329, J1231-1411, J2214+3000)

\section{INTRODUCTION}

Before the launch of the Fermi Gamma-ray Space Telescope, the only pulsars with definitive detections in $\gamma$-rays (using EGRET on the Compton Gamma Ray Observatory) were young and very energetic $\left(\dot{E}>10^{36} \mathrm{erg} \mathrm{s}^{-1}\right)$ or nearby older systems $\left(\dot{E}>10^{34} \mathrm{erg} \mathrm{s}^{-1}\right.$; Thompson 2004). A possible detection of pulsed $\gamma$-rays from the energetic millisecond pulsar (MSP) J0218+4232 (Kuiper et al. 2000) sparked interest in modeling MSP $\gamma$-ray emission (e.g., Zhang \& Cheng 2003; Harding et al. 2005) and encouraged one group (Story et al. 2007) to predict that many new MSPs might be detected in $\gamma$-rays or discovered in radio follow-up of unidentified Fermi sources.

The launch of Fermi and the extraordinary sensitivity of the Large Area Telescope (LAT; Atwood et al. 2009) confirmed those predictions of $\gamma$-ray-bright MSPs with detections of eight relatively normal radio MSPs using only the first few months of

\footnotetext{
${ }^{21}$ Current address: Naval Research Laboratory, Washington, DC 20375, USA.
}

Fermi events (Abdo et al. 2009a). Those MSPs were detected via the folding of $\gamma$-rays modulo the known spin and orbital ephemerides from radio timing campaigns (Smith et al. 2008).

In order to best utilize radio telescope time to search either for radio counterparts to new $\gamma$-ray-selected pulsars or to search blindly for radio pulsations from $\gamma$-ray sources that might contain pulsars, we formed the Pulsar Search Consortium (PSC), a group of approximately 20 LAT team members and/or pulsar experts associated with large radio telescopes around the world. This Letter describes one of the PSC's first programs, which used the Green Bank Telescope (GBT) to search 25 unassociated sources from the Fermi LAT Bright Source List (BSL; Abdo et al. 2009b).

\section{OBSERVATIONS AND DATA ANALYSIS}

We selected 25 sources from the Fermi LAT Bright Source List that were (1) unassociated with known pulsars or active galactic nuclei (AGNs), (2) unassociated with X-ray counterparts that had been previously deeply searched for radio 
pulsations (e.g., IC443; F. Camilo et al. 2011, in preparation), (3) statistically non-variable, and (4) at declinations $>-35^{\circ}$. We observed each of the sources ${ }^{22}$ for approximately $45-50$ minutes using the prime focus receiver at the GBT centered at $820 \mathrm{MHz}$ with $200 \mathrm{MHz}$ of bandwidth. The GBT pointings, all taken between 2009 July and October, were actually centered on the positions from an internal LAT source list using nine months of sky-survey data prepared in a similar fashion to the Fermi LAT First Source Catalog (i.e., "1FGL"; Abdo et al. 2010). The individual GBT pointings at $820 \mathrm{MHz}$ had FWHM $=0.25$ and covered either all or a substantial fraction of the $95 \%$ error regions for the vast majority of the sources.

We sampled the summed power from two polarizations in 2048 frequency channels with 8-bits every $61.44 \mu$ s using the GUPPI pulsar backend. ${ }^{23}$ Each pointing generated approximately $100 \mathrm{~GB}$ of data, which were recorded to hard drives for processing off-site. The $820 \mathrm{MHz}$ center frequency was chosen as a compromise between the competing effects of sky temperature (from the Galactic synchrotron background) and beam size as well as steep pulsar spectra and the effects of interstellar dispersion and scattering.

For a pulsar with a pulse width of $\sim 10 \%$ of the period, the search sensitivity was approximately $0.06 \times(29 \mathrm{~K}+$ $\left.T_{\text {sky }}\right) /(32 \mathrm{~K}) \mathrm{mJy}$, where $T_{\text {sky }}$ is the contribution at $820 \mathrm{MHz}$ of the Galactic synchrotron background. The majority of the sources (17 of them) were within three degrees of the Galactic plane where $T_{\text {sky }} \sim 12-40 \mathrm{~K}$, although two were very near the Galactic center with $T_{\text {sky }} \sim 100-150 \mathrm{~K}$. Eight of the sources were well off the Galactic plane $\left(|b|>5^{\circ}\right)$ and had $T_{\text {sky }} \sim 3-10 \mathrm{~K}$. For those sources, our search sensitivity was 0.06-0.08 mJy for normal pulsars at all reasonable dispersion measures (DMs) and MSPs up to DM $\sim 100 \mathrm{pc} \mathrm{cm}^{-3}$. In general, the observations were factors of 2-12 deeper than the best pulsar surveys that have previously covered these regions (e.g., Manchester et al. 2001; Cordes et al. 2006).

We processed the data, after de-dispersing into $~ 9000$ DMs over the range $0-1055 \mathrm{pc} \mathrm{cm}^{-3}$, using both acceleration searches (to improve sensitivity to pulsars in binary systems) and single pulse searches (to provide sensitivity to pulsars with sporadic or giant-pulse-like emission) using standard tools found in PRESTO $^{24}$ (Ransom et al. 2002). No new pulsar-like signals were found in any of the low Galactic latitude $\left(|b|<5^{\circ}\right)$ sources. However, four new MSPs were detected amid the eight high Galactic latitude sources, in OFGLs J0614.3-3330, $\mathrm{J} 1231.5-1410$, J2214.8+3002, and J2302.9+4443. The MSP in 0FGL J2302.9+4443 was detected first in an independent PSC survey by the Nançay telescope and will be reported elsewhere (Cognard et al. 2011). The rest of this Letter details the properties of the other three MSPs.

\subsection{The New MSPs}

The first two pulsars detected, J2214+3000 and J1231-1411, were undergoing substantial accelerations due to orbital motion during the discovery observations. PSR J0614-3329 was initially uncovered in an unaccelerated search, although orbital

\footnotetext{
22 The LAT Bright Sources observed with the GBT were 0FGLs J0614.33330, J1231.5-1410, J1311.9-3419, J1653.4-0200, J1741.4-3046, J1746.0-2900, J1801.6-2327, J1805.3-2138, J1814.3-1739, J1821.41444, J1834.4-0841, J1836.1-0727, J1839.0-0549, J1844.1-0335, J1848.6-0138, J1855.9+0126, J1900.0+0356, J1911.0+0905, J1923.0+1411, J2001.0+4352, J2027.5+3334, J2110.8+4608, J2214.8+3002, J2302.9+4443, and $\mathrm{J} 2339.8-0530$.

23 https://safe.nrao.edu/wiki/bin/view/CICADA/NGNPP

$24 \mathrm{http}: / /$ www.cv.nrao.edu/ sransom/presto/
}

motion was detected in the discovery observations via a more precise timing analysis. The fact that all three MSPs were in unknown binaries demanded a radio timing program to determine precise orbital parameters and constrain their astrometric positions before detailed $\gamma$-ray timing and analysis could commence.

Each MSP was observed with several different observing setups at the GBT, the Lovell Telescope at Jodrell Bank, and the Nançay radio telescope. The Arecibo telescope also observed PSR J2214+3000 several times. At the GBT, GUPPI was used with bandwidths of 100,200 , and $800 \mathrm{MHz}$ centered at 350 , 820 , and $1500 \mathrm{MHz}$, respectively. At Jodrell Bank and Nançay, observations were made with bandwidths of $200-300 \mathrm{MHz}$ centered near $1400 \mathrm{MHz}$. Standard radio timing procedures were used (Lorimer \& Kramer 2005) and the orbital parameters were fit to high precision with TEMPO2 (Hobbs et al. 2006).

After several months of radio timing and using trial X-ray positions based on point sources from Swift, XMM-Newton, and/ or Chandra, $\gamma$-ray pulsations from each MSP were detected with the LAT using orbitally demodulated events (see also Section 2.2). We determined average $\gamma$-ray pulse times of arrival (TOAs) using the maximum likelihood $\gamma$-ray timing techniques described by Ray et al. (2010) after integrating source photons modulo the predicted pulse period between 22 and 36 days per TOA. The resulting joint timing solutions, using both radio and $\gamma$-ray TOAs, as well as the derived physical parameters of the MSPs, are presented in Table 1 . The radio and $\gamma$-ray pulse profiles are shown in Figure 1.

The three new pulsars, besides being three of the brightest $\gamma$-ray MSPs in the sky, appear to be relatively normal, nearby ( $\leqslant 2 \mathrm{kpc}$ ), radio MSPs, with $\sim 3 \mathrm{~ms}$ spin periods, surface magnetic field strengths of $(2-3) \times 10^{8} \mathrm{G}$, and spin-down luminosities of $\sim 2 \times 10^{34} \mathrm{erg} \mathrm{s}^{-1}$. J0614-3329 and J1231-1411 have orbital periods of 53.6 and 1.9 days, respectively, with companions of mass $\sim 0.2-0.3 M_{\odot}$, consistent with the orbital period-white dwarf mass relation of Rappaport et al. (1995). $\mathrm{J} 2214+3000$ is a so-called black-widow system with a very low mass companion $\left(\sim 0.02 M_{\odot}\right)$ and likely timing irregularities, similar to pulsars B1957+20 (Fruchter et al. 1988), J2051-0827 (Stappers et al. 1996), and J0610-2100 (Burgay et al. 2006), and only the fourth such system known in the Galactic disk. While we currently have no evidence for radio eclipses from the pulsar (at least at frequencies $\geqslant 1.4 \mathrm{GHz}$ ), its formation was likely similar to that of those other systems (e.g., King et al. 2005).

\section{2. $\gamma$-ray Analysis}

The Fermi LAT is sensitive to $\gamma$-rays with energies 0.02-300 GeV (Atwood et al. 2009). The Fermi LAT skysurvey data set used here for spectral analysis spans from 2008 August 4 to 2010 February 4. We selected "Pass 6 Diffuse" class events-i.e., events passing the most stringent background rejection cuts-with energies above $0.1 \mathrm{GeV}$ and rejected events with zenith angles $>105^{\circ}$ to limit contamination from $\gamma$-rays from the Earth's limb. We used "Pass6 v3" instrument response functions (IRFs).

The $\gamma$-ray light curves shown in Figure 1 are constructed from events as described above, although using seven additional months of data through 2010 September 14, and with energy and radius cuts to optimize the signal to noise for each pulsar. The energy and radius cuts used for PSRs J0614-3329, $\mathrm{J} 1231-1411$, and $\mathrm{J} 2214+3000$ are (1.0, $0.35 \mathrm{GeV}),(1.2$, $0.35 \mathrm{GeV})$, and $(1.0,0.7 \mathrm{GeV})$, respectively. 
Table 1

Parameters for the New MSPs

\begin{tabular}{|c|c|c|c|}
\hline Parameter & PSR J0614-3329 & PSR J1231-1411 & PSR J2214+3000 \\
\hline Fermi BSL association (0FGL) & J0614.3-3330 & $\mathrm{J} 1231.5-1410$ & $\mathrm{~J} 2214.8+3002$ \\
\hline Fermi 1 year source (1FGL) & J0614.1-3328 & $\mathrm{J} 1231.1-1410$ & $\mathrm{~J} 2214.8+3002$ \\
\hline \multicolumn{4}{|c|}{ Timing parameters } \\
\hline Right Ascension (R.A., J2000) & $06^{\mathrm{h}} 14^{\mathrm{m}} 10^{\mathrm{s}} .3478(3)$ & $12^{\mathrm{h}} 31^{\mathrm{m}} 11^{\mathrm{s}} .3132(7)$ & $22^{\mathrm{h}} 14^{\mathrm{m}} 38^{\mathrm{s}} .8460(1)$ \\
\hline Declination (decl., J2000) & $-33^{\circ} 29^{\prime} 54^{\prime \prime} .118(4)$ & $-14^{\circ} 11^{\prime} 43^{\prime \prime} .63(2)$ & $+30^{\circ} 00^{\prime} 38^{\prime \prime} .234(4)$ \\
\hline Proper motion in R.A. $\left(\right.$ mas $\mathrm{yr}^{-1}$ ) & $\ldots$ & $-1.0(2) \times 10^{2}$ & $\ldots$ \\
\hline Proper motion in decl. $\left(\right.$ mas $\left.\mathrm{yr}^{-1}\right)$ & $\ldots$ & $-3(4) \times 10^{1}$ & $\ldots$ \\
\hline Pulsar period (ms) & $3.148669579439(9)$ & $3.683878711077(3)$ & $3.119226579079(4)$ \\
\hline Pulsar frequency $(\mathrm{Hz})$ & $317.5944552995(9)$ & $271.4530196103(2)$ & $320.5922925597(4)$ \\
\hline Frequency derivative $\left(\mathrm{Hz} \mathrm{s}^{-1}\right)$ & $-1.77(7) \times 10^{-15}$ & $-1.68(1) \times 10^{-15}$ & $-1.44(3) \times 10^{-15}$ \\
\hline Frequency 2 nd deriv. $\left(\mathrm{Hz} \mathrm{s}^{-2}\right)$ & $\ldots$ & $\ldots$ & $1.7(4) \times 10^{-23}$ \\
\hline Reference epoch (MJD) & 55100 & 55100 & 55100 \\
\hline Dispersion measure $\left(\mathrm{pc} \mathrm{cm}^{-3}\right)$ & $37.049(1)$ & $8.090(1)$ & $22.557(1)$ \\
\hline Orbital period (days) & $53.5846127(8)$ & $1.860143882(9)$ & $0.416632943(5)$ \\
\hline Projected semimajor axis (1t-s) & $27.638787(2)$ & $2.042633(3)$ & $0.0590800(9)$ \\
\hline Orbital eccentricity & $0.0001801(1)$ & $4(3) \times 10^{-6}$ & $<2 \times 10^{-4}$ \\
\hline Longitude of periastron (deg) & $15.92(4)$ & $3.2(4) \times 10^{2}$ & $\ldots$ \\
\hline Epoch of periastron (MJD) & $55146.821(7)$ & $55016.8(2)$ & $\ldots$ \\
\hline Epoch of ascending node (MJD) & $\ldots$ & $\ldots$ & $55094.137854(2)$ \\
\hline Span of timing data (MJD) & $54683-55422$ & $54683-55430$ & $54683-55415$ \\
\hline Number of $\gamma$-ray TOAs & 24 & 32 & 20 \\
\hline $\operatorname{rms} \gamma$-ray TOA residual $(\mu \mathrm{s})$ & 99.1 & 24.4 & 110.2 \\
\hline Number of radio TOAs & 328 & 136 & 437 \\
\hline rms radio TOA residual $(\mu \mathrm{s})$ & 7.1 & 9.3 & 5.0 \\
\hline \multicolumn{4}{|c|}{ Derived parameters } \\
\hline Mass function $\left(M_{\odot}\right)$ & $0.007895133(3)$ & $0.00264460(2)$ & $1.2755(1) \times 10^{-6}$ \\
\hline Min companion Mass $\left(M_{\odot}\right)$ & $\geqslant 0.28$ & $\geqslant 0.19$ & $\geqslant 0.014$ \\
\hline Galactic longitude (deg) & 240.50 & 295.53 & 86.86 \\
\hline Galactic latitude (deg) & -21.83 & 48.39 & -21.67 \\
\hline DM-derived distance $(\mathrm{kpc})$ & 1.9 & 0.4 & 1.5 \\
\hline Flux density at $820 \mathrm{MHz}(\mathrm{mJy})$ & 1.5 & 0.4 & 2.1 \\
\hline Surface magnetic field $\left(10^{8} \mathrm{G}\right)$ & 2.4 & 2.9 & 2.1 \\
\hline Characteristic age (Gyr) & 2.8 & 2.6 & 3.5 \\
\hline Spin-down lumin, $\dot{E}\left(10^{34} \mathrm{erg} \mathrm{s}^{-1}\right)$ & 2.2 & 1.8 & 1.8 \\
\hline \multicolumn{4}{|c|}{$\gamma$-ray spectral fit parameters } \\
\hline$K\left(10^{-11} \mathrm{ph} \mathrm{cm}^{-2} \mathrm{~s}^{-1} \mathrm{MeV}^{-1}\right)$ & $2.12 \pm 0.10 \pm 0.13$ & $2.62 \pm 0.16 \pm 0.18$ & $0.94 \pm 0.11 \pm 0.05$ \\
\hline Spectral index $\Gamma$ & $1.44 \pm 0.05 \pm 0.07$ & $1.40 \pm 0.07 \pm 0.05$ & $1.44 \pm 0.13 \pm 0.11$ \\
\hline$E_{\text {cutoff }}(\mathrm{GeV})$ & $4.49 \pm 0.54_{-0.84}^{+1.38}$ & $2.98 \pm 0.33_{-0.29}^{+0.43}$ & $2.53 \pm 0.50_{-0.29}^{+0.43}$ \\
\hline$F_{100}\left(10^{-8} \mathrm{ph} \mathrm{cm}^{-2} \mathrm{~s}^{-1}\right)$ & $9.52 \pm 0.46 \pm 0.45$ & $10.57 \pm 0.62 \pm 0.39$ & $3.83 \pm 0.44 \pm 0.06$ \\
\hline$G_{100}\left(10^{-11} \mathrm{erg} \mathrm{cm}^{-2} \mathrm{~s}^{-1}\right)$ & $10.86 \pm 0.35 \pm 1.06$ & $10.33 \pm 0.35 \pm 0.87$ & $3.32 \pm 0.21 \pm 0.24$ \\
\hline TS & 5270.3 & 4798.4 & 958.0 \\
\hline$\Delta \mathrm{TS}_{\text {cutoff }}$ & 184.7 & 203.3 & 63.3 \\
\hline$\underline{\eta(\%)}$ & 210 & 11 & 49 \\
\hline \multicolumn{4}{|c|}{$\mathrm{X}$-ray parameters } \\
\hline X-ray source R.A. (J2000) & $06^{\mathrm{h}} 14^{\mathrm{m}} 10^{\mathrm{s}} 3(3)$ & $12^{\mathrm{h}} 31^{\mathrm{m}} 11^{\mathrm{s}} .3(4)$ & $22^{\mathrm{h}} 14^{\mathrm{m}} 38^{\mathrm{s}} .84(3)$ \\
\hline X-ray source decl. (J2000) & $-33^{\circ} 29^{\prime} 54(5)$ & $-14^{\circ} 11^{\prime} 43(6)$ & $30^{\circ} 00^{\prime} 38^{\prime \prime} .2(6)$ \\
\hline $\mathrm{BB}$ temperature $(\mathrm{keV})$ & $0.23(5)$ & $0.21(5)$ & $0.25(4)$ \\
\hline$F_{\mathrm{BB}, 0.5-8 \mathrm{keV}}\left(10^{-14} \mathrm{erg} \mathrm{cm}^{-2} \mathrm{~s}^{-1}\right)$ & $8.7_{-3.9}^{+3.4}$ & $15_{-7.4}^{+5.3}$ & $2.9_{-0.7}^{+0.6}$ \\
\hline$L_{\mathrm{BB}, 0.5-8 \mathrm{keV}}\left(10^{30} \mathrm{erg} \mathrm{s}^{-1}\right)$ & $38_{-17}^{+15}$ & $2.9_{-1.4}^{+1.0}$ & $7.8_{-1.9}^{+1.6}$ \\
\hline
\end{tabular}

Notes. Numbers in parentheses represent $2 \sigma$ uncertainties in the last digit as determined by TEMPO2 using the DE405 Solar System Ephemeris for the timing parameters and $1 \sigma$ uncertainties for the other parameters. The time system used is Barycentric Dynamical Time (TDB). Minimum companion masses were calculated assuming a pulsar mass of $1.4 M_{\odot}$. The DM distances were estimated using the NE2001 Galactic electron density model and likely have 20\% uncertainties (Cordes \& Lazio 2002). The gamma-ray spectral parameters are from fits of exponentially cutoff power laws as described in Section 2.2. $F_{100}$ and $G_{100}$ give the integrated photon or energy flux above $0.1 \mathrm{GeV}$, respectively, while the last two parameters are gamma-ray detection significance of the source and significance of an exponential cutoff (as compared to a simple power law), where the approximate Gaussian significance is given by $\sim \sqrt{\mathrm{TS}}$, and TS is the Test Statistic TS $=2 \Delta \log$ (likelihood) between models with and without the source. The first errors are statistical and the second errors are systematic errors calculated from the bracketing IRFs. The $\gamma$ ray efficiency, $\eta=L_{\gamma} f_{\Omega} / \dot{E}=4 \pi D^{2} G_{100} / \dot{E}$, assumes a beaming correction factor, $f_{\Omega}=1$. The X-ray results are from Swift for PSRs J0614-3329 and J1231-1411, and Chandra for PSR J2214+3000. The quoted positional uncertainty for J2214+3000 is dominated by a systematic error of $0 . ' 6$ in the absolute Chandra astrometry (statistical error $=0$ '.2). X-ray fluxes and luminosities from 0.5 to $8 \mathrm{keV}$,

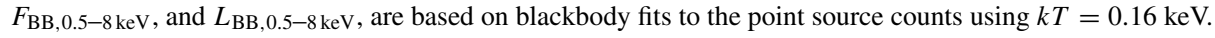




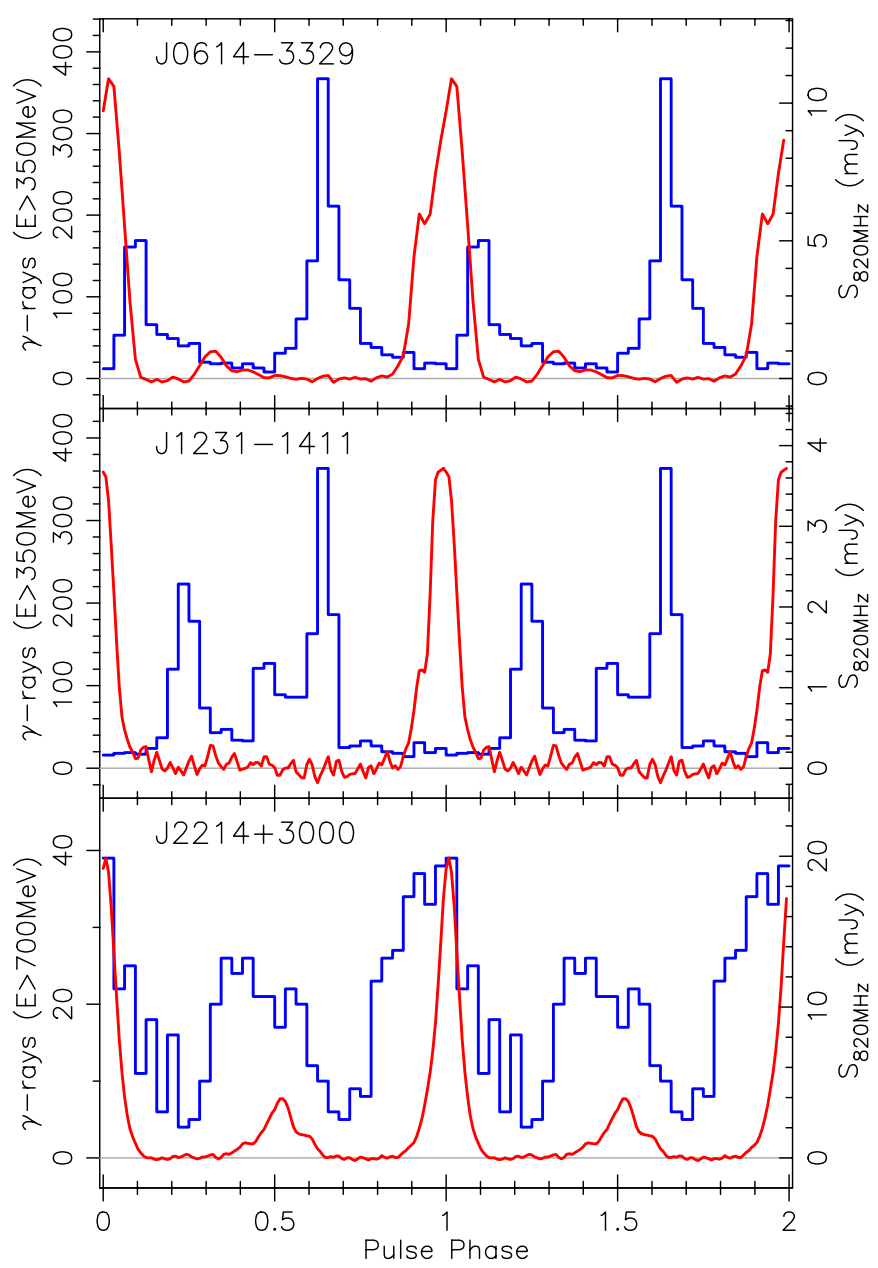

Figure 1. Radio and $\gamma$-ray pulse profiles for the three new MSPs. The red lines correspond to the $820 \mathrm{MHz}$ discovery pulse profiles from the GBT with the $820 \mathrm{MHz}$ flux density scale on the right. The blue lines are the Fermi pulse profiles with the photon counts and low energy cut used for the $\gamma$-ray selections listed on the left. There are 2043, 2341, and 621 photons in the $\gamma$-ray profiles for PSRs J0614-3329, J1231-1411, and J2214+3000, respectively.

We derived the $\gamma$-ray spectrum of each pulsar using a maximum-likelihood method implemented in the LAT Science Tool gtlike. ${ }^{25}$ We analyzed a region of $10^{\circ}$ radius centered on the radio position of each pulsar and modeled each region by including all sources from the 1FGL (Abdo et al. 2010) within $17^{\circ}$ of the pulsar along with Galactic and isotropic diffuse emission (models gll_iem_v02 and isotropic_iem_v02, respectively ${ }^{26}$ ). The power-law spectral parameters for all sources within $10^{\circ}$ of the pulsar and a normalizing scale factor for the diffuse emission spectrum were allowed to be free in the fit.

We modeled the spectrum of each MSP using a power law with an exponential cutoff where the three parameters, the differential flux $K$, the photon index $\Gamma$, and the cutoff energy $E_{\text {cutoff }}$, were allowed to vary in the fit. The phase-averaged $\gamma$ ray spectra obtained for each pulsar are shown in Figure 2 and the spectral parameters are given in Table 1 . The uncertainty in the LAT effective area is estimated to be $\leqslant 5 \%$ near $1 \mathrm{GeV}, 10 \%$ below $0.1 \mathrm{GeV}$, and $20 \%$ over $10 \mathrm{GeV}$. The resulting systematic errors on the three spectral parameters, propagated from the uncertainties on the LAT effective area, were calculated using a set of "modified IRFs" bracketing the nominal (Pass6 v3) one.

\footnotetext{
25 http://fermi.gsfc.nasa.gov/ssc/data/analysis/documentation/

26 http://fermi.gsfc.nasa.gov/ssc/data/access/lat/BackgroundModels.html
}

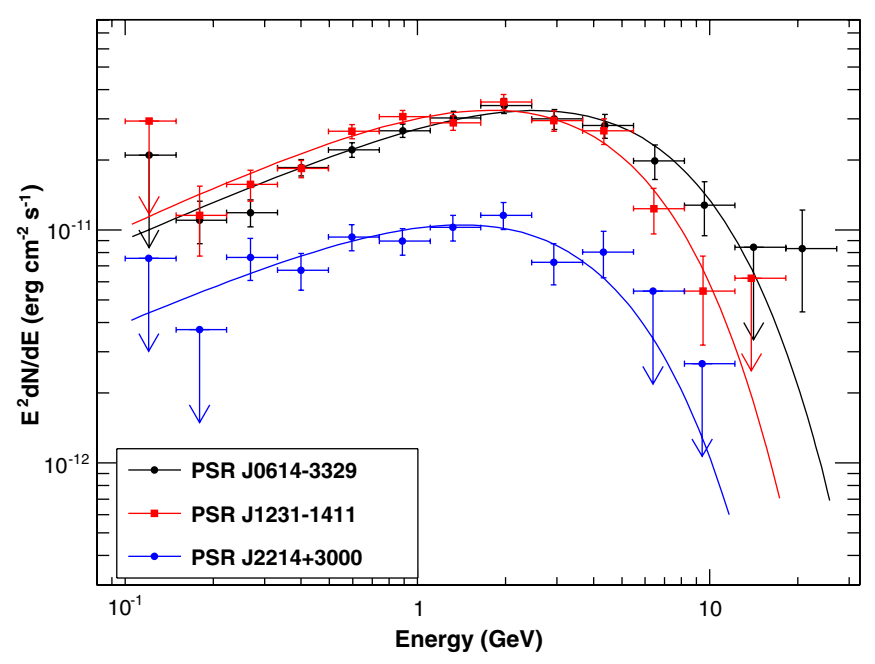

Figure 2. $\gamma$-ray spectra for the three MSPs. The flux points on the curve were obtained from independent fits in each energy bin, as explained in the text. The curves represent the spectrum fit with a simple exponentially cutoff power law in the full energy range of $0.1-100 \mathrm{GeV}$.

We verified the significance of the exponential cutoff in each spectrum with a likelihood ratio test (Mattox et al. 1996). A simple power-law model is rejected significantly for all three pulsars relative to an exponentially cutoff power law, as indicated by the test statistic $\Delta \mathrm{TS}_{\text {cutoff }}$ listed in Table 1 for the addition of one free parameter.

The flux points in Figure 2 were obtained by repeating the likelihood analysis in each energy band, assuming a powerlaw spectrum with a photon index fixed at 2 and a free flux normalization parameter for all sources.

\subsection{X-ray Analysis}

To search for X-ray counterparts, we observed the field of each pulsar with the Swift-XRT (Burrows et al. 2005) with exposures ranging from 2.6 to 15.9 ks. For J0614-3329 and J1231-1411, we detected X-ray point sources at the locations of the pulsars. In the Swift observation of PSR J2214+3000, there is no significant source at the location of the pulsar, however we also obtained a Chandra ACIS-I observation of this region and detected the pulsar with it. A detailed analysis of this Chandra observation will be presented elsewhere.

The X-ray counterparts to the MSPs are soft sources and were fit to blackbody spectra using XSPEC, fixing the absorption values to zero. The resultant temperatures for the three X-ray sources were in the range $\sim 0.21-0.25 \mathrm{keV}$, which in the case of J1231-1411, is consistent with that derived from deeper Suzaku data (Maeda et al. 2011). Allowing for additional Galactic absorption fixed to the values of $(3.4-5.7) \times 10^{20} \mathrm{~cm}^{-2}$ from Kalberla et al. (2005), we found negligible differences in the fitted parameters. The Swift and Chandra positional localizations, as well as approximate fluxes and luminosities from the blackbody fits, are in Table 1.

For PSR J1231-1411 we were able to do more detailed spectral analysis using XMM-Newton. On 2009 July 15 $X M M-N e w t o n$ observed the field of this as-yet unidentified BSL source with all three EPIC instruments. Data from each instrument were analyzed utilizing the Science Analysis System software version 10.0.0 and the calibration update of 2010 May 8. We filtered the data for bad events and excluded times of high particle background, yielding $29.4 \mathrm{ks}$ and $29.5 \mathrm{ks}$ of good time for the EPIC-MOS1 and -MOS2 instruments and $24.1 \mathrm{ks}$ 
for EPIC-PN. All three instruments utilized Full Frame mode so none had sufficient time resolution to allow searches for X-ray pulsations.

The XMM-Newton X-ray images of the J1231-1411 field reveal a relatively isolated, moderately bright, point source that we name XMMUJ123112-141146 at the best radio position of the pulsar to the accuracy of the EPIC instruments. We generated spectra of this source by extracting events from around the pulsar in $35^{\prime \prime}$ regions for the MOS images and a $25^{\prime \prime}$ region (due to a chip gap) for the PN image. This yielded spectra consisting of 853, 812 , and 2164 events from the MOS1, MOS2, and PN cameras, respectively. We group the counts with at least 30 events per spectral bin for the MOS spectra and 45 events per bin for the PN spectra using the FTOOL grppha. Background spectra were extracted from nearby regions $100^{\prime \prime}$ and $55^{\prime \prime}$ in radius from the same CCD chips for the MOS and PN instruments, respectively.

Using XSPEC (v12.6.0) we simultaneously fit the MOS1, MOS2, and PN spectra in the energy range $0.4-3.0 \mathrm{keV}$. An absorbed power-law model is formally acceptable with a reduced $\chi^{2}=0.977$ (61 dof) but with an extremely steep photon index of $\Gamma=4.23_{-0.38}^{+0.41}(90 \%$ confidence) and column density of $n_{\mathrm{H}}=\left(1.8_{-0.5}^{+0.6}\right) \times 10^{21} \mathrm{~cm}^{-2}$ (90\% confidence). Such a column density is significantly higher than that expected for this direction based on galactic surveys $\left(n_{\mathrm{H}}=3.45 \times 10^{20} \mathrm{~cm}^{-2}\right.$; Kalberla et al. 2005). A fit to these data utilizing a model of an absorbed neutron star non-magnetic hydrogen atmosphere (phabs × nsatmos; Heinke et al. 2006) with the neutron star mass and radius held fixed at $1.4 M_{\odot}$ and $10 \mathrm{~km}$, and the source distance fixed at the DM value of $0.4 \mathrm{kpc}$ yields a reduced $\chi^{2}=1.41$ (61 dof). In this model there are significant residuals above $1.5 \mathrm{keV}$ so we add a power-law component with photon index fixed at 1.8 and obtain an improved fit with $\chi^{2}=$ 1.09 (60 dof). This latter fit yields an atmospheric temperature (seen at infinity) $T_{\text {eff }}=61_{-12}^{+6} \mathrm{eV}$ (90\% confidence), a best fit $n_{\mathrm{H}}$ consistent with zero and a $90 \%$ confidence upper limit of $5 \times 10^{20} \mathrm{~cm}^{-2}$, and flux in the $0.5-3 \mathrm{keV}$ energy band $(1.15 \pm 0.05) \times 10^{-13} \mathrm{erg} \mathrm{cm}^{-2} \mathrm{~s}^{-1}$. In this model the power-law spectral component accounts for roughly $25 \%$ of the total flux in the $0.5-3 \mathrm{keV}$ energy band.

\section{CONCLUSIONS}

We have identified three new nearby radio MSPs as the counterparts of bright and previously unassociated Fermi LAT sources at high Galactic latitude. Our non-detection of young pulsars or MSPs in the more numerous sources searched at low Galactic latitude is likely due to our only moderate sensitivity improvements (typically $2-3 \times$ ) over the best surveys of those regions to date (e.g., Manchester et al. 2001) due to higher sky temperatures resulting from our lower observing frequency. Additionally, the complicated and confused nature of the Galactic plane in $\gamma$-rays makes the positive identification of point sources difficult. Several of the bright sources may be blends of other sources or the result of insufficient modeling of the Galactic background. Nonetheless, deeper surveys at frequencies of $1.5-2 \mathrm{GHz}$ of these sources may prove more fruitful in the future.

The new pulsars are very typical radio MSPs in terms of spin period, binary parameters, magnetic field strength, spin-down luminosity, and characteristic age, and their unusual brightness in $\gamma$-rays is likely due more to their proximity than to especially energetic emission processes in their magnetospheres. The very high implied $\gamma$-ray efficiency for PSR J0614-3329 suggests that it is likely closer, by up to a factor of two or more, than predicted by the NE2001 model (Cordes \& Lazio 2002). The line of sight to PSR J0614-3329 is nearly tangent to the Gum Nebula where NE2001 shows an exceptionally steep DM gradient. Additionally, the pulsar's $\gamma$-ray emission is likely not isotropic, but only covers tens of percent of the sky. These large efficiencies in general, though, are consistent with the tens of percent values found by Abdo et al. (2009a) for radio MSPs detected in $\gamma$-rays and imply that MSPs are very efficient producers of $\gamma$-rays.

We do not have proper motion measurements for pulsars J0614-3329 or J1231-1411 and so their measured spin-down rates are contaminated at some level (likely $\lesssim 10 \%$ ) by the Shklovskii effect (Shklovskii 1970). There is a statistically significant proper motion measurement of $\sim 100$ mas $\mathrm{yr}^{-1}$ for J1231-1411, though, which implies a Shklovskii effect at $400 \mathrm{pc}$ larger than the measured spin-down rate for the pulsar. If the proper motion is confirmed at this level, the requirement to have the pulsar intrinsically spinning down gives an upper limit for the pulsar's distance of $\sim 240 \mathrm{pc}$. Timing observations over the next several years will determine the proper motions and possibly the timing parallaxes for each of the pulsars.

In all three cases we identified X-ray counterparts to the pulsars which substantially aided in the rapid establishment of timing solutions. The three MSPs appear to have fairly typical X-ray properties for radio MSPs (e.g., Bogdanov et al. 2006) with primarily soft thermal-like spectra and X-ray luminosities in the $10^{30}-10^{31} \mathrm{erg} \mathrm{s}^{-1}$ range, approximately $10^{-4}$ to $10^{-3}$ of their $\gamma$-ray luminosities.

The radio flux densities of $\sim 1 \mathrm{mJy}$ near $1 \mathrm{GHz}$ are large enough to make the MSPs potentially useful for a wide variety of timing projects, such as the detection of gravitational waves via long-term pulsar timing (e.g., NANOGrav ${ }^{27}$ ), yet they are small enough to explain why earlier large-area surveys for pulsars missed them (e.g., Manchester et al. 1996; Lorimer et al. 2005). In addition, the fact that many of the nearby radio MSPs are being detected in $\gamma$-rays and vice versa argues that the sizes of the radio and $\gamma$-ray beams are comparable for MSPs (likely within a factor of $\sim 2$ ), and that deep radio and $\gamma$-ray surveys may allow us to eventually detect a large percentage of the local population of these sources. In the short term, the fact that Fermi can point us to nearby radio MSPs is already causing a large increase in the number of known systems, with much less effort than is required by sensitive large-area radio surveys. If most radio MSPs produce $\gamma$-rays as these early results seem to indicate, MSPs may contribute to the diffuse isotropic $\gamma$-ray background (Faucher-Giguère \& Loeb 2010).

We acknowledge helpful discussions with Natalie Webb and Lynne Valencic. The National Radio Astronomy Observatory is a facility of the National Science Foundation operated under cooperative agreement by Associated Universities, Inc. This work was partially supported by NASA Grant No. NNG09EE57I. The Fermi LAT Collaboration acknowledges support from a number of agencies and institutes for both development and the operation of the LAT as well as scientific data analysis. These include NASA and DOE in the US, CEA/Irfu and IN2P3/CNRS in France, ASI and INFN in Italy, MEXT, KEK, and JAXA in Japan, and the K. A. Wallenberg Foundation, the Swedish Research Council and the National Space Board in Sweden. Additional support from INAF in Italy and CNES in France for

\footnotetext{
27 http://nanograv.org
} 
science analysis during the operations phase is also gratefully acknowledged.

Facilities: GBT (GUPPI), Fermi (LAT), XMM (EPIC), CXO (ACIS), Swift (XRT)

\section{REFERENCES}

Abdo, A. A., et al. 2009a, Science, 325, 848

Abdo, A. A., et al. 2009b, ApJS, 183, 46

Abdo, A. A., et al. 2010, ApJS, 188, 405

Atwood, W. B., et al. 2009, ApJ, 697, 1071

Bogdanov, S., Grindlay, J. E., Heinke, C. O., Camilo, F., Freire, P. C. C., \& Becker, W. 2006, ApJ, 646, 1104

Burgay, M., et al. 2006, MNRAS, 368, 283

Burrows, D. N., et al. 2005, Space Sci. Rev., 120, 165

Cognard, I., et al. 2011, ApJ, submitted

Cordes, J. M., \& Lazio, T. J. W. 2002, arXiv:astro-ph/0207156

Cordes, J. M., et al. 2006, ApJ, 637, 446

Faucher-Giguère, C., \& Loeb, A. 2010, J. Cosmol. Astropart. Phys., JCAP01(2010)005

Fruchter, A. S., Stinebring, D. R., \& Taylor, J. H. 1988, Nature, 333, 237

Harding, A. K., Usov, V. V., \& Muslimov, A. G. 2005, ApJ, 622, 531

Heinke, C. O., Rybicki, G. B., Narayan, R., \& Grindlay, J. E. 2006, ApJ, 644, 1090
Hobbs, G. B., Edwards, R. T., \& Manchester, R. N. 2006, MNRAS, 369, 655

Kalberla, P. M. W., Burton, W. B., Hartmann, D., Arnal, E. M., Bajaja, E., Morras, R., \& Pöppel, W. G. L. 2005, A\&A, 440, 775

King, A. R., Beer, M. E., Rolfe, D. J., Schenker, K., \& Skipp, J. M. 2005, MNRAS, 358, 1501

Kuiper, L., Hermsen, W., Verbunt, F., Thompson, D. J., Stairs, I. H., Lyne, A. G., Strickman, M. S., \& Cusumano, G. 2000, A\&A, 359, 615

Lorimer, D. R., \& Kramer, M. 2005, Handbook of Pulsar Astronomy (Cambridge: Cambridge Univ. Press)

Lorimer, D. R., et al. 2005, MNRAS, 359, 1524

Maeda, K., et al. 2011, ApJ, submitted

Manchester, R. N., et al. 1996, MNRAS, 279, 1235

Manchester, R. N., et al. 2001, MNRAS, 328, 17

Mattox, J. R., et al. 1996, ApJ, 461, 396

Ransom, S. M., Eikenberry, S. S., \& Middleditch, J. 2002, AJ, 124, 1788

Rappaport, S., Podsiadlowski, P., Joss, P. C., DiStefano, R., \& Han, Z. 1995, MNRAS, 273, 731

Ray, P. S., et al. 2010, ApJ, submitted (arXiv:1011.2468)

Shklovskii, I. S. 1970, SvA, 13, 562

Smith, D. A., et al. 2008, A\&A, 492, 923

Stappers, B. W., et al. 1996, ApJ, 465, L119

Story, S. A., Gonthier, P. L., \& Harding, A. K. 2007, ApJ, 671, 713

Thompson, D. J. 2004, in Cosmic Gamma-Ray Sources, ed. K. S. Cheng \& G. E. Romero (Astrophysics and Space Science Library, Vol. 304; Dordrecht: Kluwer), 149

Zhang, L., \& Cheng, K. S. 2003, A\&A, 398, 639 